\title{
CORRELACIÓN ENTRE EL GRADO DE HIPERTENSIÓN ARTERIALY EL ÍNDICE DE ESTRÉS OXIDATIVO. ESTUDIO DE COHORTE EN UNA POBLACIÓN DE PACIENTES HIPERTENSOS SISTÉMICOS EN REPÚBLICA DOMINICANA
}

\author{
Correlation between the arterial hypertension degree and oxidative stress \\ index. Cohort study in a population of systemic hypertensive patients in \\ Dominican Republic
}

\author{
R. Núñez-Musa ${ }^{1 *}$, A. J. Núñez-Sellés ${ }^{2 * *}$, W. Mañón Rossi ${ }^{1 * * *}$, R. Guillén Marmolejos ${ }^{1 * * * *}$, \\ G. Martínez-Sánchez ${ }^{3 \Omega}$
}

Recibido: mayo 16, 2019 • Aprobado: junio 24, 2019

Cómo citar: Núñez-Musa R, Núñez-Sellés AJ, Mañón Rossi W, Marmolejos RG, Martínez-Sánchez G. Correlación entre el grado de hipertensión arterial y el índice de estrés oxidativo. Estudio de cohorte en una población de pacientes hipertensos sistémicos en República Dominicana. cysa [Internet]. 25 de julio de 2019 [citado 26 de julio de 2019];3(2): 17-33. Disponible en:

https://revistas.intec.edu.do/index.php/cisa/article/view/1475

\section{Resumen}

Introducción: la hipertensión arterial (HTA) es una de las mayores cargas de enfermedad y riesgo para infarto cardíaco, la insuficiencia cardíaca y el fallo renal. Se reconoce que el estrés oxidativo (EO) es un determinante en el desarrollo de complicaciones y el progreso de la HTA. Se determinó el índice de EO (IEO) en individuos con HTA y en un grupo sano control, para evaluar su posible correlación.

Materiales y métodos: se midió IEO en una población de 112 individuos con HTA de distintos grados entre 50 y 70 ańos escogidos al azar y se comparó con los valores de un grupo control de voluntarios sanos, con la intención de definir el grado de correlación entre los niveles del IEO y la severidad de HTA, mediante la medición de biomarcadores para el EO en lisado de eritrocitos.

1. Contract Research Organization CREODR, Ave. Gustavo Mejía Ricart No. 273, Edif. CORA 2, 5to Nivel, La Castellana, Santo Domingo, República Dominicana

2. Universidad Nacional Evangélica (UNEV), Paseo de los Periodistas 54, Miraflores, Santo Domingo, Distrito Nacional, República Dominicana

3. Consultor Independiente, Ancona, Italy

\begin{abstract}
Introduction: Arterial hypertension (AHT) is one of the major burdens of disease and risk for cardiac infarction, heart failure and renal failure. It is recognized that the oxidative stress (OS) is a determining factor in the development of complications and the progress of the AHT. OS Index (OSI) in individuals with AHT and a healthy control group, was determined to assess their possible correlation
\end{abstract}

Methods: OSI was measured in a population of 112 individuals with AHT of different levels between 50 and 70 years old, chosen at random and compared with the values of healthy volunteers control group with the aim of defining the degree of correlation between the levels of the OSI and the AHT severity, by measuring biomarkers for OS in a red cell lysate.

\footnotetext{
Correo-e de los autores:

*rnunezmusa@creodr.com

** nunez500412@gmail.com

***wmanonrossi@gmail.com

**** rafaelgui218@gmail.com

$\Omega$ gregorcuba@yahoo.it
} 
Resultados: a pesar de que los beneficios de la terapia antioxidante (TAO) no han sido definitivamente probadas, en gran parte porque las enfermedades complejas no dependen de un solo componente fisiopatogénico, el EO sigue siendo una piedra angular en el desarrollo de complicaciones y el empeoramiento de los cuadros clínicos de muchos padecimientos. La demostración de biomarcardores específicos mejora la posibilidad de una TAO dirigida. El presente ensayo demostró que la edad, el género y la etnia no influyen en el IEO y que el EO fue severo en los casos de HTA III, moderado en HTA il y estuvo ausente en el subgrupo con HTA grado I.

Conclusiones: estos resultados sugieren una relación entre los niveles de EO y severidad de HTA y sustenta evidencias para diseñar nuevos ensayos clínicos que evalúen la eficacia de una TAO adyuvante en el manejo de la HTA.

Palabras clave: índice de estrés oxidativo; biomarcadores de redox; daño oxidativo; terapia antioxidante; hipertensión.

\section{Introducción}

La hipertensión arterial (HTA) es una de las mayores cargas de enfermedad en el mundo y constituye un alto riesgo para otras condiciones patológicas de impacto como el infarto cardíaco, la insuficiencia cardíaca, sobre todo crónica, lesiones valvulares y aneurisma aórtico, así como para el fallo renal. No es rara la asociación de La HTA con enfermedades metabólicas, con lo cual el pronóstico de riesgo y muerte es más severo. La relación de estrés oxidativo (EO) y HTA ha sido ampliamente documentada y ha sido propuesta, a veces, como parte de los procesos fisiopatogénicos de la enfermedad ${ }^{1,2}$. En estudios recientes, tanto la reducción de la superóxido dismutasa y la glutatión-peroxidasa como la elevación de peróxido de hidrógeno y otros productos de la peroxidación lipídica, han sido identificadas en casos de HTA de grados variables de severidad y complicaciones ${ }^{3}$. El EO severo presente en distintas patologías, incluida la HTA, parece relacionarse también con el curso y las complicaciones ${ }^{4-6}$, lo que sugiere que la adición de antioxidantes en la dieta pudiera ser beneficiosa, una vez conocidos los mecanismos principalmente deteriorados para instalar
Discussion: Despite the benefits of an antioxidant therapy (AOT) have not been definitely proven, largely because the complex diseases do not depend on a single pathophysiological component, OS remains as a cornerstone in the development of complications and the worsening of the clinical pictures of many ailments. The demonstration of specific biomarkers improve the possibility of an addressed AOT. This trial showed th at the age, gender and ethnicity do not influence the OSI and that OS was severe in HTA III cases, moderate in HTA II cases and was absent in the subgroup with HTA I.

Conclusions: These results suggest a relationship between levels of EO and severity of hypertension and support evidence to design new clinical trials assessing the efficacy of an adjuvant AOT in the management of HTA.

Keywords: Oxidative stress index; redox biomarkers; oxidative damage; antioxidant therapy; hypertension.

la terapia antioxidante (TAO) correspondiente.

Ya algunos estudios han mostrado la posibilidad de que una TAO dirigida pueda contribuir favorablemente en el pronóstico de enfermedades y complicaciones metabólicas y cardiovasculares ${ }^{7-10}$, aunque hasta el momento no hay mucha investigación sobre la relevancia de la medición de los biomarcadores del EO como potenciales determinantes, individualmente o no, del pronóstico de la HTA o como indicadores de la instauración de una TAO coadyuvante.

A pesar de que los estudios sobre los beneficios de la TAO no han sido concluyentes, en gran parte porque las enfermedades complejas no dependen de un solo componente fisiopatogénico, el EO sigue siendo una piedra angular en el desarrollo de complicaciones y el empeoramiento de los cuadros clínicos de muchos padecimientos ${ }^{4-6,11-14}$ y la TAO constituye un recurso potencialmente útil para controlar o revertir muchos de estos efectos ${ }^{15-17}$, especialmente en $\mathrm{HTA}^{3,18,19}$.

Otro aspecto en el fallo de los estudios para demostrar la utilidad de los antioxidantes, puede corresponder 
al método inadecuado para medir el EO del paciente, en particular, para determinar su estado redox ${ }^{20}$. La mayoría de los estudios no muestra una selección de los biomarcadores de EO específicamente relacionados con la HTA, ni evalúa el sustrato fisiológico más apropiado ${ }^{21,22}$.

La elección de los biomarcadores adecuados para cuantificar el IEO con la finalidad de instalar una terapia coadyuvante en la HTA es de vital importancia, ya que su eficacia dependerá del sustrato fisiológico al cual van dirigidos los productos antioxidantes. Esto es debido a que los biomarcadores pueden revelar información determinante que permite direccionar la terapia en forma eficaz, como es la de conocer cuáles productos de la lípido-peroxidación están presentes, cuáles son los compuestos resultantes de las modificaciones proteicas, qué actividades de las enzimas antioxidantes están alteradas y, en el aspecto epigenético, qué modificaciones del $\mathrm{ADN}$ que alteran la expresión genética son identificables ${ }^{23-25}$. No obstante estas evidencias, la mayor parte de los datos publicados sobre el uso de una TAO en HTA no señalan cuáles biomarcadores están involucrados en el proceso en estudio. Este factor de sesgo puede ser eliminado mediante la determinación del IEO a través de la medición de distintos biomarcadores y relacionarlos con su valor clínico y su relevancia en la evolución de las enfermedades ${ }^{29}$.

En 2017, Núñez et al., patentaron una técnica para la determinación del IEO y correlacionar la evolución de la enfermedad con los valores del EO, mediante la aplicación de una escala porcentual que toma como referencia una población sana de voluntarios ${ }^{30}$.

El presente reporte muestra los resultados de la determinación del IEO en una cohorte de individuos con HTA de distintos grados, comparada con un grupo sano control, con el propósito de evaluar la posible correlación entre el IEO y el grado de la HTA y demostrar el beneficio potencial de agregar una TAO coadyuvante al tratamiento estándar de la HTA.

\section{Materiales y métodos}

\section{Diseño del ensayo}

La encuesta EFRICARD II ${ }^{31}$ identificó 286,350 sujetos con HTA en la provincia de Santo Domingo, para un $34.7 \%$ de prevalencia. En 2014 se reportó un $34 \%$ de muertes asociadas a enfermedades cardiovasculares, en las que la HTA fue concurrente en más del $33 \%$ de los casos $^{32}$. Para este estudio prospectivo, doble ciego, se eligieron sujetos con diagnóstico de HTA en tratamiento para evaluar su IEO, en instituciones de salud de la ciudad de Santo Domingo, República Dominicana, entre 50 y 70 ańos de edad, dentro de cuyo rango se encuentra el $30 \%$ de la población nacional ${ }^{33}$.

El protocolo de ensayo clínico fue aprobado por la Comisión Nacional de Bioética en Salud (CONABIOS) bajo el número 008-2016. Todos los sujetos fueron reclutados entre mayo y agosto de 2016 y leyeron, comprendieron y completaron el "Acta de Consentimiento Informado", según los lineamientos nacionales y los establecidos en la Declaración de Helsinki de 2013.

Toda la información de los sujetos de estudio fue mantenida en confidencialidad y un sistema de códigos se creó para permitir su identificación y el manejo del expediente médico y las muestras.

\section{Selección de la muestra}

El tamaño de la muestra fue determinado mediante la aplicación en línea XLSTAT-Biomed para muestreo estadístico, tomando de base la incidencia de HTA para la población etaria objeto de estudio y acorde con el reporte de EFRICARD $\mathrm{II}^{32}$. Se conformaron dos grupos y tres subgrupos, según se describe en la tabla 1, para obtener niveles de significación estadística $(\alpha)=0.05$, potencia $(1-\beta)=0.2$, desviación estándar $(\sigma)=2$, y detectar diferencias $(\mathrm{d})=1$. El total de sujetos reclutados fue de 235 . 
Tabla 1. Edad promedio y género de la población estudiada

\begin{tabular}{|l|c|c|c|c|}
\hline \multicolumn{1}{|c|}{ Grupo } & Masculinos & Femeninos & Total & Edad promedio \\
\hline Grupo I (Control) & $\mathbf{5 1}$ & $\mathbf{7 2}$ & $\mathbf{1 2 3}$ & $\mathbf{6 7 . 0} \pm \mathbf{6 . 0}$ \\
\hline Grupo II (HTA) & $\mathbf{4 5}$ & $\mathbf{6 5}$ & $\mathbf{1 1 2}$ & $\mathbf{6 9 . 4} \pm \mathbf{6 . 6}$ \\
\hline Subgrupo IIa (HTA I) & 20 & 30 & 50 & $67.9 \pm 5.0$ \\
\hline Subgrupo IIb (HTA II) & 15 & 21 & 36 & $70.4 \pm 5.8$ \\
\hline Subgrupo IIC (HTA III) & 12 & 14 & 26 & $71.0 \pm 5.5$ \\
\hline TOTAL & $\mathbf{9 8}$ & $\mathbf{1 3 7}$ & $\mathbf{2 3 5}$ & $\mathbf{6 8 . 1} \pm \mathbf{6 . 2}$ \\
\hline
\end{tabular}

Fuente: elaboración propia.

\section{Leyenda:}

HTA: Hipertensión Arterial.

No hubo diferencia significativa entre los grupos etarios ( $p>0.05)$.

\section{Criterios de inclusión}

1. Pacientes de ambos géneros con edades entre 50 y 70 años, con diagnóstico confirmado de HTA de la provincia de Santo Domingo.

2. Historia de evolución de la enfermedad de dos años o más.

3. No fumadores.

4. No consumo habitual de bebidas alcohólicas o sustancias estupefacientes.

5. No consumo de antioxidantes en los últimos doce meses.

\section{Criterios de exclusión}

1. Otras enfermedades degenerativas concomitantes.

2. Consumo habitual de bebidas alcohólicas o sustancias estupefacientes o de antioxidantes antes y durante el estudio.

3. Estar participando en otro ensayo clínico.

Todos los sujetos fueron evaluados integralmente por un especialista y el grado de hipertensión fue calculado según las "Guías prácticas de 2013” de la ESC y ESH ${ }^{34}$ como HTA grado I: niveles de presión sistólica (PS) 140-159 mm Hg y presión diastólica (PD) de 90-99 mmHg; grado II: PS 160-179 y PD 100-109; grado III: PS $\geq 180$ y PD $\geq 110$. Estas informaciones, así como los datos personales y las listas de inclusión y de exclusión, se remitieron al investigador principal para ser incorporadas al "Cuaderno de registro de datos" bajo un código único.

\section{Procesamiento de muestras}

Se tomó una muestra de $100 \mu \mathrm{L}$ de sangre a cada sujeto mediante punción digital con una lanceta estéril y de inmediato fue traspasada a un tubo de Eppendorf con $2 \mathrm{ml}$ de solución salina normal. Las muestras se mantuvieron a $8^{\circ} \mathrm{C}$ hasta su transporte al laboratorio para pruebas analíticas dentro de las 24 horas de la toma. Fueron centrifugadas a 3,000 g, el supernadante se eliminó y $2 \mathrm{ml}$ de agua doblemente destilada fue ańadida a la fracción eritrocitaria para inducir su hemólisis. Una fracción de $200 \mu \mathrm{L}$ fue colectada con una micropipeta, llevada al tubo de Eppendorf $(1 \mathrm{ml})$ y mantenida a $-10^{\circ} \mathrm{C}$ en un refrigerador vertical para su procesamiento a lo largo de 60 días. 


\section{Técnicas de laboratorio}

Potencial de Peroxidación (PP)

- Prueba para evaluar la susceptibilidad individual a la peroxidación.

- Se determinó aplicando una técnica modificada según Ozdemirler et al., ${ }^{35}$. El lisado de eritrocitos (LE) se incubó con sulfato de cobre II $(2 \mathrm{mM})$ a $37^{\circ} \mathrm{C}$ por $24 \mathrm{~h}$. Se determinó la absorbancia a $586 \mathrm{~nm}$ en tiempo 0 y 24 h después de la incubación. La diferencia en absorbancia constituyó el PP. Los valores se llevaron a una escala normalizada de 100 unidades. Cada muestra fue analizada por triplicado y los resultados expresados como $(\bar{Y}) \pm$ SD.

\section{Estatus Antioxidante Total (EAT)}

- El EAT se define como la suma total de todos los antioxidantes endógenos y los derivados de los alimentos presentes en el líquido extracelular de un individuo ${ }^{36}$. La interacción entre todos ellos provee protección contra las Especies Reactivas de Oxígeno (ERO). Se determinó a través de la cinética de los radicales libres de la reacción con el catión 2,2'-azinobis-(3-etilbenzotiazolina-6-ácido sulfónico (ABTS+) a $600 \mathrm{~nm}$ de acuerdo a $\mathrm{Ozcan}^{37}$. Los valores se llevaron a una escala normalizada de 100 unidades. Cada muestra fue analizada por triplicado y los resultados expresados como $(\overline{\mathrm{Y}}) \pm \mathrm{SD}$.

\section{Capacidad Antioxidante Equivalente al Trolox (TEAC)}

- Mide la capacidad antioxidante total de las biomoléculas, basada en la conversión de ABTS+ oxidado a ABTS vía transferencia simple de electrones o de átomos de hidrógeno y usa como referencia la actividad del Trolox.

- Se determinó mediante una técnica modificada por Blois ${ }^{38}$. El LE se incubó con una solución amortiguadora de acetato sódico $(40 \mathrm{mM}, \mathrm{pH}$ = 5.5) y $1 \mathrm{~mL}$ de etanol por una hora a $37^{\circ} \mathrm{C}$.
Se añadió $1 \mathrm{~mL}$ de 1,1-difenil-2-picrilhidrazil (DFPH) $100 \mu \mathrm{M}$ después de la incubación a una parte de la muestra incubada y la absorbancia a $517 \mathrm{~nm}$ fue definida para ambas alícuotas (con y sin DFPH). La diferencia entre las absorbancias se expresó a escala de 100 unidades. Cada muestra se procesó en triplicado y los resultados se expresaron como $(\overline{\mathrm{Y}}) \pm \mathrm{SD}$.

\section{Prueba de Malondialdehido (MDA)}

- El MDA es un importante biomarcador de la peroxidación lipídica presente en el EO.

- Las concentraciones de MDA se midieron de acuerdo al método de Esterbauer y Cheeseman ${ }^{39}$. El LE se analizó con el kit LPO-586 (Calbiochem, La Jolla, CA, EE. UU.). La producción de un cromóforo estable, después de 40 minutos de incubación a $45^{\circ} \mathrm{C}$, fue cuantificada a $586 \mathrm{~nm}$. Para la estandarización, soluciones recién preparadas de MDA-bis[dimetilacetal] fueron utilizadas y cuantificadas bajo condiciones idénticas. Los valores experimentales fueron normalizados a una escala de 100 unidades. Cada muestra se analizó por triplicado y los resultados se expresaron como $(\bar{Y}) \pm$ SD.

\section{Grupos Carbonilos (GC)}

- En los procesos de daño oxidativo a proteínas, algunos aminoácidos como lisina, prolina y arginina, se oxidan dando lugar a grupos carbonilo, de modo que el contenido en carbonilos de las proteínas se puede emplear como un indicador de daño oxidativo.

- Los GC se determinaron de acuerdo a Oliver et al., ${ }^{40}$. El LE fue suspendido en $1 \mathrm{~mL}$ de ácido clorhídrico (2 M) y $1 \mathrm{~mL}$ de 2,4-dinitrofenilhidrazina $(0.2 \%)$ e incubado con agitación a $37^{\circ} \mathrm{C}$ durante 1 hora. Se añadió $1 \mathrm{~mL}$ de ácido tricloroacético $(10 \%)$ y el precipitado fue extraído con $10 \mathrm{~mL}$ de una mezcla de etanol-etilacetato (1:1). El sedimento fue reprecipitado con ácido tricloroacético (10\%). Una muestra control de 
proteína (albúmina de huevo) fue disuelta en $1 \mathrm{~mL}$ de hidrocloruro de guanidina en solución amortiguadora fosfato potásico $(20 \mathrm{mM}, \mathrm{pH}=6.5)$. Tanto la muestra como el control se centrifugaron y la absorbancia se determinó a un intervalo de 370 y $375 \mathrm{~nm}$. La diferencia entre los valores de absorbancia se llevó a escala de 100 unidades. Cada muestra se analizó por triplicado y los resultados se expresaron como $(\bar{Y}) \pm S D$.

\section{Grupos Sulfhidrilo (GS)}

- Los GS sirven como donadores de electrones y su proporción está relacionada con la concentración de glutatión. El glutatión juega un papel esencial en el sistema redox intracelular.

- Los GS se determinaron de acuerdo al método de Sedlak y Lindsay ${ }^{41}$ con el reactivo de Ellman. El LE fue disuelto en $1 \mathrm{~mL}$ de solución amortiguadora de fosfato potásico $(50 \mathrm{mM}, \mathrm{pH}=7.5)$ y centrifugado. La solución sobrenadante se hizo reaccionar con el reactivo de Ellman y la absorbancia fue determinada a $600 \mathrm{~nm}$. El mismo procedimiento se llevó a cabo para la muestra control de proteína no oxidada (albúmina de huevo). La diferencia entre las absorbancias se expresó en escalas de 100 unidades. Cada muestra se analizó por triplicado y los resultados se expresaron como $(\bar{Y}) \pm \mathrm{SD}$.

\section{Superóxido Dismutasa (SOD)}

- La SOD cataliza la dismutación de superóxido en oxígeno y peróxido de hidrógeno, como mecanismo antioxidante para la mayoría de las células.

- Su actividad fue determinada según lo descrito por Del Mestro y McDonald ${ }^{42}$. El LE fue mezclado con $2.8 \mathrm{~mL}$ de una solución amortiguadora de TRIS [tris(hidroximetil) aminometano], (0.05M, pH = 8.2), $100 \mu \mathrm{L}$ de agua destilada y $50 \mu \mathrm{L}$ de una solución de EDTA $(1 \mathrm{mM})$. Se agregaron $50 \mu \mathrm{L}$ de una solución de pirogalol $(0.124 \mathrm{M})$ y la absorbancia fue determinada a $420 \mathrm{~nm}$ después de $10 \mathrm{~s}$ y cada $10 \mathrm{~s}$ por un minuto (6 lecturas). El mismo procedimiento fue aplicado a una preparación en blanco sin muestra de LE. La diferencia de absorbancia fue calculada tanto para la muestra de estudio como para el blanco y la actividad del SOD fue determinada. Esta diferencia fue expresada en escalas de 100 unidades. Cada muestra se analizó por triplicado y los resultados se expresaron como $(\bar{Y}) \pm$ SD.

\section{Catalasa (CAT)}

- La CAT descompone el peróxido de hidrógeno $\left(\mathrm{H}_{2} \mathrm{O}_{2}\right)$, que es un potente agente oxidante, en agua y $\mathrm{O}_{2}$. Es una enzima de mucha importancia para la protección celular contra el daño oxidativo causado por las ERO.

- Su actividad fue determinada siguiendo el método de Haining y Legan ${ }^{43}$. El LE fue mezclado en una celda de cuarzo con $0.9 \mathrm{~mL}$ de solución amortiguadora de fosfato potásico $(\mathrm{pH}=7)$ y $500 \mu \mathrm{L}$ de una solución de $\mathrm{H}_{2} \mathrm{O}_{2}(0.05 \mathrm{M})$ en solución amortiguadora. Se determinó la absorbancia a $240 \mathrm{~nm}$ en los tiempos 10 y $70 \mathrm{~s}$. Se determinó también la absorbancia del blanco con $1.5 \mathrm{~mL}$ de solución amortiguadora. La diferencia de absorbancias fue calculada para ambas muestras y la actividad de la CAT fue determinada. Esta diferencia fue expresada en escalas de 100 unidades. Cada muestra se analizó por triplicado y los resultados se expresaron como $(\bar{Y}) \pm \mathrm{SD}$

\section{Glutatión-Peroxidasas (GPx)}

- La GPx es una familia de enzimas para la protección celular contra el EO. Son responsables de convertir los peróxidos a sus correspondientes alcoholes y para ello emplean como cofactor el glutatión reducido (GSH) que se transforma en glutatión oxidado (GSSG).

- La actividad de las GPx se determinó basada en la experiencia de Gil et al., ${ }^{44}$. La GPx cataliza la 
reducción del sustrato hidroperóxido de cumeno y oxida el GSH. En presencia de glutatión reductasa y NADPH el GSSG es convertido inmediatamente a su forma reducida con una oxidación paralela de NADPH a NADP+. La disminución en la absorbancia a $340 \mathrm{~nm}$ fue medida. Los resultados se expresaron como $\mathrm{U} / \mathrm{g} \mathrm{Hb}$. La actividad de GPx se expresó en escalas de 100 unidades. Cada muestra se analizó por triplicado y los resultados se expresaron como $(\bar{Y}) \pm S D$.

Todos los reactivos y solventes fueron adquiridos en Sigma Aldrich (EE. UU.) o JT Baker (EE. UU.), a menos que se especifique lo contrario. Los valores de absorbancia fueron determinados con un Genesys 10S, (Thermo Scientific, EE. UU.), con celdas de cuarzo de $1 \mathrm{~cm}$ de profundidad; la incubación se realizó en placas de Petri descartables (Fisherbrand, EE. UU.) en una incubadora DNI-150 (MRC, Israel). Las muestras se centrifugaron en una centrífuga MiniSpin (Eppendorf, Alemania); las muestras de sangre se extrajeron con AutoLancets (Palcolabs, EE. UU.) y se colectaron en tubos PVC de 0.5-, 1.0 y $2.0 \mathrm{~mL}$ (Eppendorf, Alemania).

\section{Procesamiento de datos}

Todos los datos generados a partir de los resultados de las técnicas analíticas de laboratorio se tabularon independientemente para cada subgrupo y para el grupo control del estudio. El IEO se calculó siguiendo la siguiente fórmula:

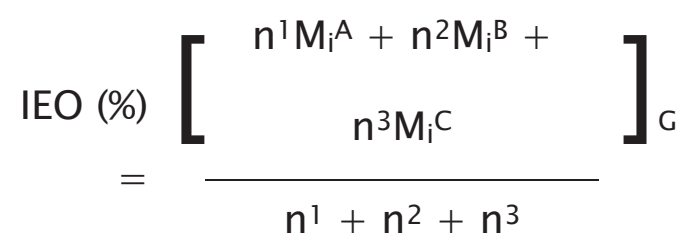

donde:

$$
\mathrm{M}^{\mathrm{A}}=\frac{\Sigma T_{A} \text { DM/HTA }}{\Sigma T_{A} \text { SANOS }} \cdot 100
$$

$$
\begin{gathered}
\mathrm{M}_{\mathrm{i}}^{\mathrm{B}}=\frac{\sum T_{B}^{\text {DM } / H T A}}{\sum T_{B}^{\text {SANOS }}} \cdot 100 \\
\mathrm{M}_{\mathrm{i}}^{\mathrm{C}}=\frac{\sum T_{C^{\text {DM } / H T A}}}{\sum T_{C}^{\text {SANOS }}} \cdot 100
\end{gathered}
$$

donde:

$\mathrm{n}^{1}=3$ (número de biomarcadores para determinar la concentración total de radicales libres: PP, EAT y TEAC);

$\mathrm{n}^{2}=3$ (número de biomarcadores específicos del daño oxidativo celular/tisular: MDA, GC, GS);

$\mathrm{n}^{3}=3$ (número de biomarcadores específicos de las enzimas endógenas para la defensa antioxidante: SOD, CAT, GPx);

$\mathrm{G}=$ género $(\mathrm{M}$ o $\mathrm{F})$ y grupo etario $(1: 60-64 ; 2$ : $\geq 65$ años de edad).

Toda la data experimental fue procesada por dos técnicos estadísticos independientes, que generaron tablas en duplicado y desconocían los resultados uno del otro. La información obtenida en los grupos y subgrupos fueron analizados mediante el programa estadístico SPSS 9.0. Se hicieron pruebas no paramétricas de Friedman para algunos subgrupos relacionados y para cambios dentro de grupos y subgrupos se usó el test pareado de Wilconxon. La prueba de Mann-Whitney U se usó para estimar las diferencias estadísticas $(\mathrm{p}<0.05)$ entre los subgrupos. Los resultados fueron reportados como valores medios \pm media de desviación estándar (MDE).

El Grado de Daño Oxidativo (GDO) se calculó como el porcentaje de IEO determinado en los subgrupos versus el IEO obtenido en los voluntarios sanos de acuerdo a la tabla 2 . 
Tabla 2. Valores cuantitativos y cualitativos en la escala del Grado de Daño Oxidativo según los valores del Índice de Estrés Oxidativo

\begin{tabular}{|c|c|}
\hline IEO & GDO \\
\hline 0 & Ninguno \\
\hline$<30$ & Ligero \\
\hline $30-60$ & Moderado \\
\hline$>60$ & Severo \\
\hline
\end{tabular}

Fuente: elaboración propia.

\section{Leyenda:}

IEO: Índice de Estrés Oxidativo; GDO: Grado de Daño Oxidativo

\section{Resultados y discusión}

Los resultados de la determinación del IEO en el En la tabla 5 se describe la determinación del GDO grupo i (control) y en el grupo II (HTA), se muesen el grupo II. tran en las tablas 3 y 4 , respectivamente.

Tabla 3. Caracterización del Índice de Estrés Oxidativo en el grupo I (control)

\begin{tabular}{|c|c|c|c|c|c|}
\hline Género & Rangos de edad & Término $\mathbf{M}_{\mathbf{i}}^{\mathbf{A}}$ & Término $\mathbf{M}_{\mathbf{i}}^{\mathbf{B}}$ & ${\text { Término } \mathbf{M}_{\mathbf{i}}{ }^{\mathbf{C}}} \mathbf{G D O} \pm \mathbf{S D}(\mathbf{p}<\mathbf{0 . 0 5})$ \\
\hline \multirow{2}{*}{$\mathbf{M}$} & $60-64$ & $11.0 \pm 2.3$ & $10.8 \pm 1.3$ & $9.0 \pm 1.3$ & $30.7 \pm 3.3$ \\
\cline { 2 - 5 } & $\geq 65$ & $14.0 \pm 2.4$ & $14.7 \pm 1.9$ & $11.5 \pm 1.6$ & $40.2 \pm 5.7 \mathrm{ab}$ \\
\hline \multirow{2}{*}{$\mathbf{F}$} & $60-64$ & $10.7 \pm 1.6$ & $10.8 \pm 1.9$ & $9.2 \pm 1.4$ & $30.7 \pm 5.4$ \\
\cline { 2 - 6 } & $\geq 65$ & $13.3 \pm 1.2$ & $14.6 \pm 2.0$ & $12.1 \pm 1.5$ & $40.0 \pm 4.8 \mathrm{a}$ \\
\hline
\end{tabular}

Fuente: elaboración propia.

\section{Leyenda:}

Diferentes letras en superíndice significan diferencia estadística $(\mathrm{p}<0.05)$ entre las mimas series.

GDO: Grado de Daño Oxidativo; $\mathrm{M}_{\mathrm{i}}^{\mathrm{A}}=\mathrm{A}_{1}(\mathrm{PP})+\mathrm{A}_{2}($ EAT $)+\mathrm{A}_{3}\left(\right.$ TEAC); $\mathrm{M}_{\mathrm{i}}^{\mathrm{B}}=\mathrm{B}_{1}(\mathrm{MDA})+\mathrm{B}_{2}(\mathrm{GC})+\mathrm{B}_{3}(\mathrm{GS}): \mathrm{M}_{\mathrm{i}}^{\mathrm{C}}$ $=\mathrm{C}_{1}(\mathrm{SOD})+\mathrm{C}_{2}(\mathrm{CAT})+\mathrm{C}_{3}(\mathrm{GPx}) ;$ PP: Potencial de Peroxidación, TAS: Estatus Antioxidante Total, TEAC: Capacidad Antioxidante Equivalente al Trolox, GS: Grupo Sulfihidrilo; MDA: Malondialdehído, SOD: Superóxido Dismutasa, CAT: Catalasa, GPx: Glutatión Peroxidasas; M: masculinos; F: femeninos

Tabla 4. Determinación del Índice de Estrés Oxidativo en el grupo II y subgrupos a, b y c

\begin{tabular}{|c|c|c|c|c|c|}
\hline Género & Rangos de edad & Término $\mathbf{M}_{\mathrm{i}}^{\mathrm{A}}$ & Término $\mathrm{M}_{\mathrm{i}}^{\mathrm{B}}$ & Término $\mathrm{M}_{\mathrm{i}}^{\mathrm{C}}$ & $\mathrm{IEO} \pm \mathrm{SD}(\mathrm{p}<0.05)$ \\
\hline \multicolumn{6}{|c|}{ HTA II (Subgrupo IIa) } \\
\hline \multirow{2}{*}{$\mathbf{M}$} & $60-64 y$ & $9.7 \pm 1.0$ & $10.3 \pm 1.3$ & $8.7 \pm 1.4$ & $-5.8 \pm 12.4$ \\
\hline & $\geq 65 \mathrm{y}$ & $12.9 \pm 0.8 \mathrm{a}$ & $12.6 \pm 1.3$ & $11.4 \pm 1.8$ & $-9.7 \pm 12.3$ \\
\hline \multirow{2}{*}{$\mathbf{F}$} & $60-64 y$ & $9.5 \pm 0.9$ & $10.3 \pm 1.2$ & $8.9 \pm 1.5$ & $-6.2 \pm 12.8$ \\
\hline & $\geq 65 y$ & $14.3 \pm 1.0 \mathrm{ab}$ & $14.0 \pm 1.4 \mathrm{a}$ & $13.3 \pm 1.4 \mathrm{a}$ & $6.4 \pm 7.8$ \\
\hline
\end{tabular}


Correlación entre el grado de hipertensión arterial y el índice de estrés oxidativo. Estudio de cohorte en una población de pacientes hipertensos sistémicos en República Dominicana

\begin{tabular}{|c|c|c|c|c|c|}
\hline Género & Rangos de edad & Término $\mathrm{M}_{\mathrm{i}}^{\mathrm{A}}$ & Término $\mathrm{M}_{\mathrm{i}}^{\mathrm{B}}$ & Término $\mathrm{M}_{\mathrm{i}}^{\mathrm{C}}$ & $\mathrm{IEO} \pm \mathrm{SD}(\mathrm{p}<0.05)$ \\
\hline \multicolumn{6}{|c|}{ HTA II (Subgrupo IIb) } \\
\hline \multirow{2}{*}{$\mathbf{M}$} & $60-64 y$ & $25.7 \pm 2.7$ & $26.7 \pm 2.6$ & $19.2 \pm 3.1$ & $56.6 \pm 5.3 \mathrm{abc}$ \\
\hline & $\geq 65 y$ & $29.8 \pm 2.4$ & $31.1 \pm 2.1$ & $23.1 \pm 3.2$ & $51.8 \pm 4.5 \mathrm{abc}$ \\
\hline \multirow{2}{*}{$\mathbf{F}$} & $60-64 y$ & $25.4 \pm 2.3$ & $26.6 \pm 1.7$ & $18.5 \pm 2.9$ & $56.0 \pm 4.5 \mathrm{abc}$ \\
\hline & $\geq 65 y$ & $38.7 \pm 1.9 \mathrm{a}$ & $42.5 \pm 2.1 \mathrm{a}$ & $36.3 \pm 3.1 \mathrm{a}$ & $65.9 \pm 2.0 \mathrm{abc}$ \\
\hline Género & Rangos de edad & Término $\mathrm{M}_{\mathrm{i}}^{\mathrm{A}}$ & Término $\mathrm{M}_{\mathrm{i}}^{\mathrm{B}}$ & Término $\mathrm{M}_{\mathrm{i}}^{\mathrm{C}}$ & $\mathrm{IEO} \pm \mathrm{SD}(\mathrm{p}<0.05)$ \\
\hline \multicolumn{6}{|c|}{ HTA III (Subgrupo IIc) } \\
\hline \multirow{2}{*}{$\mathbf{M}$} & $60-64 y$ & $31.7 \pm 2.3$ & $35.3 \pm 1.9$ & $29.0 \pm 2.7$ & $67.9 \pm 2.3 \mathrm{abc}$ \\
\hline & $\geq 65 y$ & $39.3 \pm 1.8 \mathrm{a}$ & $43.6 \pm 1.2$ & $37.5 \pm 1.2 \mathrm{a}$ & $66.5 \pm 1.2 \mathrm{abc}$ \\
\hline \multirow{2}{*}{$\mathbf{F}$} & $60-64 y$ & $31.6 \pm 2.6$ & $35.1 \pm 1.8$ & $28.3 \pm 2.0$ & $67.5 \pm 2.4 \mathrm{abc}$ \\
\hline & $\geq 65 y$ & $40.6 \pm 2.4 \mathrm{a}$ & $46.0 \pm 2.4 \mathrm{ab}$ & $43.1 \pm 2.6 \mathrm{ab}$ & $69.1 \pm 1.8 \mathrm{abc}$ \\
\hline
\end{tabular}

Fuente: elaboración propia.

\section{Leyenda:}

Diferentes letras en superíndice significan diferencia estadística $(\mathrm{p}<0.05)$ entre las mimas series.

GDO: Grado de Daño Oxidativo; $\mathrm{M}_{\mathrm{i}}^{\mathrm{A}}=\mathrm{A}_{1}(\mathrm{PP})+\mathrm{A}_{2}(\mathrm{TAS})+\mathrm{A}_{3}(\mathrm{TEAC}) ; \mathrm{M}_{\mathrm{i}}^{\mathrm{B}}=\mathrm{B}_{1}(\mathrm{MDA})+\mathrm{B}_{2}(\mathrm{GC})+\mathrm{B}_{3}(\mathrm{GS}) ; \mathrm{M}_{\mathrm{i}}^{\mathrm{C}}=\mathrm{C}_{1}$ $(\mathrm{SOD})+\mathrm{C}_{2}(\mathrm{CAT})+\mathrm{C}_{3}(\mathrm{GPx})$; PP: Potencial de Peroxidación, TAS: Estatus Antioxidante Total, TEAC: Capacidad Antioxidante Equivalente al Trolox, GS: Grupo Sulfihidrilo; MDA: Malondialdehído, SOD: Superóxido Dismutasa, CAT: Catalasa, GPx: Glutatión Peroxidasas.

M: masculinos; F: femeninos

Tabla 5. Determinación de Grado de Daño Oxidativo en el grupo a, b y c según el Índice de Estrés Oxidativo en el grupo II

\begin{tabular}{|c|l|l|l|l|}
\hline \multirow{2}{*}{ Género } & \multicolumn{2}{|c|}{ Rangos de edad } & \multicolumn{2}{|c|}{ IEO } \\
\cline { 3 - 5 } & & \multicolumn{1}{|c|}{ Subgrupo IIa (HTA I) } & Subgrupo IIb (HTA II) & \multicolumn{1}{c|}{ Subgrupo IIc (HTA III) } \\
\hline \multirow{2}{*}{$\mathbf{M}$} & $60-64 \mathrm{y}$ & $-5.8 \pm 12.4$ & $56.6 \pm 5.3 \mathrm{abc}$ & $67.9 \pm 2.3 \mathrm{abc}$ \\
\cline { 2 - 5 } & $\geq 65 \mathrm{y}$ & $-9.7 \pm 12.3$ & $51.8 \pm 4.5 \mathrm{abc}$ & $66.5 \pm 1.2 \mathrm{abc}$ \\
\cline { 2 - 5 } & GDO & NINGUNO & MODERADO & SEVERO \\
\hline \multirow{3}{*}{ F } & $60-64 \mathrm{y}$ & $-6.2 \pm 12.8$ & $56.0 \pm 4.5 \mathrm{abc}$ & $67.5 \pm 2.4 \mathrm{abc}$ \\
\cline { 2 - 5 } & $\geq 65 \mathrm{y}$ & $6.4 \pm 7.8$ & $65.9 \pm 2.0 \mathrm{abc}$ & $69.1 \pm 1.8 \mathrm{abc}$ \\
\cline { 2 - 5 } & GDO & NINGUNO & MODERADO & SEVERO \\
\hline
\end{tabular}

Fuente: elaboración propia.

Leyenda:

IEO: Índice de Estrés Oxidativo; GDO: Grado de Daño Oxidativo; HTA: Hipertensión Arterial. 
Grupo I (control)

La edad, etnia y distribución por género en ambos grupos fueron comparables: $68.1 \pm 6.2$ años de edad en promedio. La población universo fue predominantemente negra y mestiza $(41.4 \pm 9.1$ y $32.2 \pm 8.2 \%$, respectivamente) en el $70 \%$ de los grupos en estudio, siendo el género femenino el de mayor presencia $(59.7 \%)$, como se puede ver en la tabla 6 .

Tabla 6. Composición étnica de los grupos del estudio

\begin{tabular}{|l|l|l|l|l|l|l|l|l|l|}
\hline \multicolumn{1}{|c|}{ Grupo } & Blancos & $\mathbf{\%}$ & \multicolumn{1}{c|}{ Negros } & $\mathbf{\%}$ & \multicolumn{1}{c|}{ Mestizos } & \multicolumn{1}{c|}{ Asiáticos } & \multicolumn{1}{c|}{ Total } \\
\hline Grupo I (Contro) & $\mathbf{2 3}$ & $\mathbf{1 8 . 7}$ & $\mathbf{6 1}$ & $\mathbf{4 9 . 6}$ & $\mathbf{3 7}$ & $\mathbf{3 0 . 1}$ & $\mathbf{2}$ & $\mathbf{1 . 6}$ & $\mathbf{1 2 3}$ \\
\hline Grupo II (HTA) & $\mathbf{3 3}$ & $\mathbf{2 9 . 5}$ & $\mathbf{4 8}$ & $\mathbf{4 2 . 9}$ & $\mathbf{3 0}$ & $\mathbf{2 6 . 8}$ & $\mathbf{1}$ & $\mathbf{0 . 8}$ & $\mathbf{1 1 2}$ \\
\hline Subgrupo IIa (HTA I) & 12 & 24.0 & 22 & 44.0 & 16 & 32.0 & 0 & 0 & 50 \\
\hline Subgrupo IIb (HTA II) & 14 & 38.9 & 17 & 47.2 & 4 & 11.1 & 1 & 2.8 & 36 \\
\hline Subgrupo IIc (HTA III) & 7 & 26.9 & 9 & 34.6 & 10 & 38.5 & 0 & 0 & 26 \\
\hline TOTAL & $\mathbf{5 6}$ & $\mathbf{2 3 . 8}$ & $\mathbf{1 0 9}$ & $\mathbf{4 6 . 4}$ & $\mathbf{6 7}$ & $\mathbf{2 8 . 5}$ & $\mathbf{3}$ & $\mathbf{1 . 3}$ & $\mathbf{2 3 5}$ \\
\hline
\end{tabular}

Fuente: elaboración propia.

\section{Leyenda:}

HTA: Hipertensión Arterial.

No hubo diferencia significativa entre los grupos étnicos ( $\mathrm{p}>0.05)$.

Los altos valores de IEO, que estuvieron entre 30 y 40 sobre una escala de 100 en ambos géneros en el grupo I, pueden explicarse, en parte, por la edad. Aunque algunos autores han descartado el efecto de las ERO en el proceso de envejecimiento o con la ocurrencia de enfermedades de la edad avanzada $^{45-47}$, otros autores y estudios recientes han retomado el tema desde otro ángulo y comprobado una relación más que eventual entre el fenómeno oxidativo exagerado y el envejecimiento y sus complicaciones ${ }^{48-51}$, aunque no sea visto como factor exclusivo o único. Por tanto, esta población control, en promedio mayor de 60 años, por razones de edad puede tener más altos IEO que otras poblaciones menores, aun en ausencia de enfermedad aparente. No hubo diferencia significativa $(\mathrm{p}>0.05)$ en IEO entre las etnias.

Estos resultados epidemiológicos, que por primera vez han examinado el daño oxidativo en una población dominicana sana mayor de 60 años, comprendieron nueve biomarcadores e incluyó no solo la sobreproducción de radicales libres (Término A), sino también el daño oxidativo biomolecular (Término B) y la actividad de las enzimas relacionadas con el mecanismo de defensa contra la oxidación (Término C). Estudios previos han enfatizado la importancia de este tipo de ensayos para correlacionar el EO y la evolución de una enfermedad adecuadamente tratada, midiendo un grupo de biomarcadores y elucidando cómo el estatus del sistema redox influye en la salud humana ${ }^{52,53}$. Estos han sido los factores que se utilizaron para determinar el IEO en pacientes con HTA mediante la comparación de sus grados de $\mathrm{EO}$ con el grupo control sano.

Grupo II (HTA)

La HTA ha sido reconocida como el mayor contribuyente del riesgo cardiovascular, siendo la principal causa de morbimortalidad en el mundo y el factor aislado de riesgo a la salud más prevalente ${ }^{54}$. Es una 
enfermedad de alta prevalencia mundial, incluida la República Dominicana (RD). El EFRICARD $2012^{31}$ reportó una prevalencia del $35 \%$ en RD. La multiplicidad de afecciones que acompañan la historia natural de la enfermedad requiere de un abordaje multidirigido basado en la incorporación de terapias combinadas que controlen, cuando no eviten, las complicaciones que derivan del progreso de la HTA, sobre todo en los casos moderadamente severos a severos del padecimiento ${ }^{20,55}$.

La HTA es un problema creciente de salud mundial y afecta a 1.13 millardos de personas ${ }^{57}$. El control sigue siendo subóptimo, con índices tan altos como del $40 \%$ o más de los pacientes hipertensos ${ }^{58}$.

Ha sido señalado que las ERO juegan un papel determinante en la fisiopatología de la $\mathrm{HTA}^{9}$, lo que bien puede asociar el desconocimiento de este impacto y su potencial relación con el fracaso para alcanzar mejores controles de los valores de presión arterial.

Los resultados del subgrupo ira revelaron que no hubo $\mathrm{EO}$ en esos pacientes. El EO de este sub-grupo fue más bajo, incluso que el del grupo control, lo cual indicó que la terapia para la HTA pudo ejercer un efecto antioxidante beneficioso (tabla 6). La mayoría de los pacientes del sub-grupo IIa tuvo terapia combinada de un bloqueador de angiotensina II con una estatina, a los cuales se les reconocen efectos antioxidantes ${ }^{59,60}$. Adicionalmente, en HTA grado I la actividad enzimática antioxidante endógena no está disminuida, por tanto, los cambios no son críticos $^{61}$.

Las ERO tienen una función en la regulación vascular a través de vías de señalización redox-sensitivas, mediante mecanismos de retroalimentación. En la HTA el EO deteriora la función endotelial, promueve la remodelación microvascular y provoca la inflamación hasta llevar a daño vascular. Estudios de laboratorio han mostrado un rol causal del EO en el daño vascular en la $\mathrm{HTA}^{62}$, pero los datos en humanos no han sido convincentes. Algunos autores estiman que esto se debe a métodos no óptimos para hacer una evaluación precisa del estado redox ${ }^{20}$. En el caso de los subgrupos irb y IIc se encontraron niveles moderados y severos del GDO, respectivamente, lo cual sugiere que el aumento del IEO lleva a mayores grados de severidad de la HTA. Si estos pacientes son candidatos para la TAO, será materia de un nuevo estudio dirigido a valorar la pertinencia o beneficio de su aplicación en cohortes con mayor seguimiento longitudinal y con una mejor estratificación de acuerdo a la historia de su enfermedad.

Algunos reportes desalentadores sobre el uso de TAO individuales o de "amplio espectro" con el empleo de vitaminas C, E y A ( $\beta$-caroteno) para ayudar en la reducción de la presión arterial ${ }^{63-68}$, han limitado la oportunidad de su uso como adyuvante en el tratamiento de la HTA, a pesar de algunos metanálisis que parecen sustentarlo ${ }^{3}$.

Dado que actualmente no está claro a cuáles mecanismos debe dirigirse la terapia antioxidante $y$, por tanto, qué tipo de compuestos son los más idóneos para componer cócteles o definir monoterapias que aumenten la eficacia del tratamiento estándar de la $\mathrm{HTA}^{69}$, el presente estudio abre la posibilidad de que, identificando con precisión qué subyace molecularmente en el curso de la enfermedad de los pacientes de una cohorte, sea aplicada una terapia de intervención a los causales del trastorno redox basada en antioxidantes.

La falta de eficacia de la TAO coadyuvante para disminuir la presión arterial en casos de HTA ha sido destacada por algunos autores ${ }^{70}$, no obstante, en otros reportes se ha documentado que una dieta rica en antioxidantes es beneficiosa para reducir el riesgo cardiovascular y controlar la HTA ${ }^{21,22}$. Existen otros factores concurrentes con roles determinantes en la patogénesis de las enfermedades crónicas que pueden ser modificados con intervenciones dirigidas a reducir el $\mathrm{EO}$, dentro de las cuales la HTA es una candidata, tomando en cuenta que la HTA: i. con frecuencia se asocia a problemas metabólicos ${ }^{71}$; ii. posee un fuerte componente 
microvascular oxidativo que la exacerba y que incrementa el dańo a órganos blanco y la mortalidad ${ }^{72,73}$; y iii. lleva a complicaciones en distintos órganos en los cuales también los fenómenos oxidativos tienen un impacto importante $e^{4,74,75}$.

Formulaciones que combinan tres o más antioxidantes son consideradas como beneficiosas para el soporte terapéutico de la HTA $^{18}$, lo cual puede ser razonable porque las dietas balanceadas y enriquecidas con una amplia variedad de frutas y vegetales, igualmente incluidas en las recomendaciones alimentarias de las guías para el manejo de la HTA, son altamente ventajosas para mejorar el estado de muchos pacientes hipertensos ${ }^{8}$. Por tanto, una terapia complementaria contra la disfunción endotelial, eje de la mayor parte de la patogenia de la HTA, que pueda interferir con el daño celular/tisular mediado por el EO, parece ser una recomendación segura, promisoria y estratégica.

La experiencia de este estudio subraya la necesidad de decidir una aplicación correcta de las TAO que resulten efectivas por la elección adecuada y específica del antioxidante, no solo para revertir o controlar los cambios fisiopatológicos involucrados en el desarrollo de la HTA, sino, y sobre todo, para prevenir sus complicaciones o mitigar, cuando no detener, la evolución desfavorable de la enfermedad. El curso de otras patologías se ha visto mejorado o potencialmente mejorado con el uso de antioxidantes específicos, con lo cual se han logrado mejores pronósticos por la reducción de las complicaciones $^{76,77}$, de donde, el pronóstico de la HTA puede igualmente verse mejorado en casos seleccionados. $^{7-10}$

Todo lo anterior implica que las complicaciones derivadas de la HTA y el desencadenamiento de EO no controlado, relacionado a este padecimiento, pueden ser prevenidas o mitigadas con la aplicación oportuna de una TAO coadyuvante a la terapia estándar, mucho mejor si esta se decide sobre la base de compuestos específicamente útiles para el problema.

\section{Conclusiones}

Las evidencias obtenidas en este estudio conducen a la hipótesis de que con el uso de métodos diagnósticos, apropiados, del EO, se puede optimizar la intervención terapéutica para decidir la formulación más efectiva de antioxidantes como tratamiento adyuvante de la HTA. La determinación del IEO en la HTA (grados II y III), en el contexto de un protocolo de ensayo clínico bien diseñado y conducido, provee el soporte necesario para el uso adecuado de la TAO en esta enfermedad, que ayude a mejorar la eficacia del tratamiento convencional y a disminuir las comorbilidades. En el presente, y a pesar de seguir siendo una materia de amplio debate, el uso agregado de antioxidantes a los tratamientos antihipertensivos ha ido progresivamente en aumento, lo que parece ser la forma más efectiva y segura de mejorar el pronóstico cardiovascular de estos pacientes, gracias a que el complejo mecanismo molecular sobre el cual descansa la desregulación de la función endotelial y el redox pueden controlarse o revertirse con el uso de productos antioxidantes específicos. Si se reconoce que la TAO puede ser un recurso viable, confiable y efectivo para enfrentar las complicaciones o frenar el deterioro de los pacientes con HTA, este estudio clínico permite avanzar en la dirección de la prevención secundaria y plantea que el uso de la TAO en HTA grado I no es de utilidad, mientras lo es para las formas más severas de la enfermedad (grados II y III).

\section{Reconocimientos}

El soporte financiero del Proyecto FONDOCYT 2012-2013-2A1-58, del Ministerio de Educación Superior, Ciencia y Tecnología de la República Dominicana, es altamente agradecido.

R. Silverio, D.A. Terrero, y M. Beras por su apoyo en el reclutamiento de casos en los hospitales.

D. Jackson, L. Kelly, J.M. Jiménez, L. Pérez por su asistencia en el reclutamiento de los voluntarios sanos. 
M. Butler y P. Santana por el soporte logístico en la Facultad de Ciencias de la Salud, de la Universidad Nacional Evangélica (UNEV).

\section{Conflicto de intereses}

Los autores del estudio no tienen conflictos de intereses ni dualidad relevante que declarar.

\section{Bibliografía}

1. Montezano AC, Touyz RM. Oxidative stress, Noxs, and hypertension: experimental evidence and clinical controversies. Annals of medicine. 2012;44(sup1): S2-16. doi: $10.3109 / 07853890.2011 .653393$.

2. Rodrigo R, González J, Paoletto F. The role of oxidative stress in the pathophysiology of hypertension. Hypertens Res. 2011;34(4): 431-40. doi: $10.1038 / \mathrm{hr} .2010 .264$.

3. Baradaran A, Nasri H, Rafieian-Kopaei M. Oxidative stress and hypertension: Possibility of hypertension therapy with antioxidants. J Res Med Sci. 2014; 19(4): 358-67.

4. Delbosc S, Paizanis E, Magous R, Araiz C, Dimo T, Cristol JP, Cros G, Azay J. Involvement of oxidative stress and NADPH oxidase activation in the development of cardiovascular complications in a model of insulin resistance, the fructose-fed rat. Atherosclerosis. 2005;179(1): 43-9.doi: https:// doi.org/10.1016/j.atherosclerosis.2004.10.018.

5. Niedowicz DM, Daleke DL. The role of oxidative stress in diabetic complications. Cell biochemistry and biophysics. 2005;43(2): 289-330. doi: https://doi.org/10.1385/CBB:43:2:289.

6. GuzikTJ,TouyzRM. Oxidativestress, inflammation, and vascular aging in hypertension. Hypertension. 2017;70(4): 660-7. doi: https://doi.org/10.1161/ HYPERTENSIONAHA.117.07802.
7. Vella RK, Pullen C, Coulson FR, Fenning AS. Resveratrol prevents cardiovascular complications in the SHR/STZ rat by reductions in oxidative stress and inflammation. BioMed research international. 2015;2015: ID 918123. doi: http://dx.doi.org/10.1155/2015/918123.

8. Lau YS, Ling WC, Dharmani Murugan MR. Boldine ameliorates vascular oxidative stress and endothelial dysfunction: Therapeutic implication for hypertension and diabetes. J Cardiovasc Pharmacol. 2015;65(6): 522-31. doi: 10.1097/ FJC.0000000000000185.

9. Sinha N, Kumar Dabla P. Oxidative stress and antioxidants in hypertension-a current review. Curr hypertens Rev. 2015;11(2):132-42. doi: 10 .2174/1573402111666150529130922.

10. Ceriello A, Testa R, Genovese S. Clinical implications of oxidative stress and potential role of natural antioxidants in diabetic vascular complications. Nutr Metab Cardiovasc Dis. 2016;26(4): 285-92. doi: 10.1016/j.numecd.2016.01.006.

11. Pourvali K, Abbasi M, Mottaghi A. Role of superoxide dismutase 2 gene Ala16Val polymorphism and total antioxidant capacity in diabetes and its complications. Avicenna J Med Biotechnol. 2016;8(2): 48-56.

12. Mikhak B, Hunter D J, Spiegelman D, Platz E A, Wu K, Erdman J W Jr, Giovannucci E. Manganese superoxide dismutase (MnSOD) gene polymorphism, interactions with carotenoid levels and prostate cancer risk. Carcinogenesis. 2008;29(12): 2335-40. doi:10.1093/carcin/bgn212.

13. Martins Gregório B, Benchimol De Souza D, Amorim de Morais Nascimento F, Matta L, Fernandes-Santos C. The potential role of antioxidants in metabolic syndrome. Current pharmaceutical design. 2016;22(7): 859-69. doi: https://doi.org/10.1155/2017/3279061.

14. Putri AY, Thaha M. Role of oxidative stress on chronic kidney disease progression. Acta Med Indones. 2016;46(3): 244-52. 
15. Velusamy T, Panneerselvam AS, Purushottam M, Anusuyadevi M, Pal PK, Jain S, et al. Protective effect of antioxidants on neuronal dysfunction and plasticity in Huntington's disease. Oxidative medicine and cellular longevity. 2017;2017: ID 3279061. doi: https://doi. org/10.1155/2017/3279061.

16. Zhang YJ, Gan RY, Li S, Zhou Y, Li AN, Xu DP, Li HB. Antioxidant phytochemicals for the prevention and treatment of chronic diseases. Molecules. 2015;20(12): 21138-56. doi: https:// doi.org/10.3390/molecules201219753.

17. Oyenihi AB, Ayeleso AO, Mukwevho E, Masola B. Antioxidant strategies in the management of diabetic neuropathy. BioMed Res Int. 2015;2015: ID 515042. doi: http://dx.doi. org/10.1155/2015/515042.

18. Ahmad KA, Yuan Yuan D, Nawaz W, Ze H, Zhuo CX, Talal B, et al. Antioxidant therapy for management of oxidative stress induced hypertension. Free Radic Res. 2017;51(4): 428-38. doi: 10.1080/10715762.2017.1322205.

19. Maiese K. New insights for oxidative stress and diabetes mellitus. Oxid Med Cell Longev. 2015;2015: ID 875961. doi: 10.1155/2015/875961.

20. Montezano AC, Dulak-Lis M, Tsiropoulou S, Harvey A, Briones AM, Touyz RM. Oxidative stress and human hypertension: vascular mechanisms, biomarkers, and novel therapies. Can J Cardiol. 2015;31(5): 631-41. doi: 10.1016/j. cjca.2015.02.008.

21. Steinhubl SR. Why have antioxidants failed in clinical trials? Am J Cardiol, 2008;101(10): S14-S19. doi: https://doi.org/10.1016/j. amjcard.2008.02.003.

22. Stephens JW, Khanolkar MP, Bain SC. The biological relevance and measurement of plasma markers of oxidative stress in diabetes and cardiovascular disease. Atheroscl. 2009; 202(2): 321-29. doi:10.1016/j.atherosclerosis.2008.06.006.
23. Ho E, Galougahi KK, Chia-Chi L, Bhindi R. Biological markers of oxidative stress: Applications to cardiovascular research and practice. Redox Biol. 2013;1: 483-91. doi: 10.1016/j.redox.2013.07.006.

24. Mañon-Rossi W, Garrido G, Nuñez-Selles AJ. Biomarkers of oxidative stress in antioxidant therapy. J Pharm Pharmacogn Res. 2016;4(2): 62-83.

25. Niu Y, DesMarais TL, Tong Z, Yao Y, Costa M. Oxidative stress alters global histone modification and DNA methylation. Free Radic Biol Med. 2015;82: 22-8. doi: https://doi.org/ 10.1016/j.freeradbiomed.2015.01.028.

26. Firuzi O, Miri R, Tavakkoli M, Saso L. Antioxidant therapy: Current status and future prospects. Curr Med Chem. 2011;18(25): 3871-88. doi: 10.2174/092986711803414368.

27. Tiwari BK, Pandey KB, Abidi AB, Rizvi SI. Markers of oxidative stress during Diabetes Mellitus. J Biomarkers. 2013; 2013: ID 378790. doi: doi.org/10.1155/2013/378790, 8 pp.

28. Frijhoff J, Winyard PG, Zarkovic N, Davies SS, Stocker R, Cheng D, et al. Clinical relevance of biomarkers of oxidative stress. Antioxid Redox Signal. 2015;23(14): 1144-70. doi: https://doi. org/10.1089/ars.2015.6317.

29. Marrocco I, Altieri F, Peluso I. Measurement and clinical significance of biomarkers of oxidative stress in humans. Oxid Med Cell Long. 2017; 2017: ID 6501046. doi: doi. or/10.1155/2017/6501046.

30. Nuñez Selles AJ, Martinez G, Mañon Rossi W. Method for determining Oxidative Stress Index in patients with Diabetes Mellitus and Arterial Hypertension. Oficina Nacional de la Propiedad Industrial (ONAPI), República Dominicana, 2017; P2017-54, 32 pp. 
31. Pichardo R, González A, Ramírez W, Escaño F, Jiménez R. Revista Española de Cardiología [Internet]. Revespcardiol.org. 2012 [citado 6 de mayo 2019]. Disponible en: https://tinyurl. com/y5mj4vp7.

32. Organización Panamericana de la Salud. República Dominicana [Internet]. Health in the Americas 2017. 2014 [citado 6 de mayo 2019]. Disponible en: https://tinyurl.com/y4jprc9h.

33. Oficina Nacional de Estadísticas. Ix Censo Nacional de Población y Vivienda 2010 (Book, 2012) [WorldCat.org] [Internet]. Worldcat.org. 2012 [citado 6 de mayo 2019]. Disponible en: https://tinyurl.com/y6ps82yb.

34. Grupo de Trabajo para el manejo de la hipertensión arterial de la Sociedad Europea de Hipertensión (ESH) y la Sociedad Europea de Cardiología (ESC). Guía de práctica clínica de la ESH/ESC 2013 para el manejo de la hipertensión arterial [Internet]. Hipertension.cl. 2013 [citado 6 de mayo 2019]. Disponible en: https://tinyurl.com/y52xs3cv.

35. Ozdemirler G, Mehmetcik G, Oztezcan S, Toker G, Sivas A, Uysal M. Peroxidation potential and antioxidant activity of serum in patients with diabetes mellitus and myocardial infarction. Horm Metab Res. 1995;27(4): 194-6.

36. Miller N, Rice-Evans C, Davies MJ. A novel method for measuring antioxidant capacity and its application to monitoring the antioxidant status in premature neonates. Clin Sci. 1993;84(4): 407-12. doi: 10.1042/cs0840407.

37. Ozcan E. A novel automated direct measurement method for total antioxidant capacity using a new generation, more stable ABTS cation. Clin Biochem. 2004;37(4): 277-85. doi: https://doi. org/10.1016/j.clinbiochem.2003.11.015

38. Blois M. Antioxidant determinations by the use of a stable free radical. Nature. 1958;181: 1199-00. doi: 10.1038/1811199a0.
39. Esterbauer H, Cheeseman KH, Determination of aldehydic lipid peroxidation products: malonaldehyde and 4-hydroxynonenal. Methods Enzymol. 1990;186: 407-21. doi: https://doi. org/10.1016/0076-6879(90)86134-H.

40. Oliver CN, Ahn B, Moerman EJ, Goldstein S, Stadtman ER. Age related changes in oxidised protein. J Biol Chem. 1987;262(12): 5488-91.

41. Sedlak J, Lindsay RH. Estimation of total protein bond and non-protein sulfhydryl group with Ellman's reagent. Anal Biochem. 1968;25: 192-205.

42. Del Mestro R, McDonald W. Oxidative enzymes in tissue homogenates. En: Greenwald R, ed., CRC Handbook for Oxygen Radical Research. Boca Raton, Florida: CRC Press; 1985. pp. 291-6.

43. Haining JL, Legan JS. Improved assay for catalase based upon steady-state substrate concentration. Anal Biochem. 1972;45(2): 469-79. doi: https:// doi.org/10.1016/0003-2697(72)90209-6.

44. Gil L, Martínez G, González I, Tarinas A, Álvarez A, Giuliani A, et al. Contribution to characterization of oxidative stress in HIV/AIDS patients, Pharmacol Res. 2003; 47(3): 217-224. https:// doi.org/10.1016/S1043-6618(02)00320-1.

45. Liochev SI. Reactive oxygen species and the free radical theory of aging Free Radic Biol Med. 2013;60:1-4. doi: 10.1016/j.freeradbiomed.

46. Lewis KN, Andziak B, Yang T, Buffenstein R. The naked mole-rat response to oxidative stress: just deal with it. Antioxid. Redox Signal. 2013;19(12): 1388-99. doi:10.1089/ars.2012.4911.

47. Holzerová E, Prokisch H. Mitochondria: Much ado about nothing? How dangerous is reactive oxygen species production? Int J Biochem Cell Biol. 2015;63: 16-20. doi: 10.1016/j. biocel.2015.01.021. 
48. Sas K, Szabó E, Vécsei L. Mitochondria, Oxidative Stress and the Kynurenine System, with a Focus on Ageing and Neuroprotection. Molecules. 2018;23(1). pii: E191. doi: 10.3390/ molecules23010191.

49. Tan J, Xu X, Tong Z, Yu Q, Lin Y, Kuang W. Decreased osteogenesis of adult mesenchymal stem cells by reactive oxygen species under cyclic stretch: a possible mechanism of age related osteoporosis. Bone Res. 2015;3: ID 15003. doi: 10.1038/boneres.2015.3.

50. Nita M, Grzybowski A. The role of the reactive oxygen species and oxidative stress in the pathomechanism of the age-related ocular diseases and other pathologies of the anterior and posterior eye segments in adults. Oxid Med Cell Longev. 2016;2016: ID 3164734. doi: $10.1155 / 2016 / 3164734$.

51. Birch-Machin MA, Bowman A. Oxidative stress and ageing. Br J Dermatol. 2016 Oct;175 Suppl 2: 26-9. doi: 10.1111/bjd.14906.

52. Block G, Dietrich M, Norkus EP, Morrow JD, Hudes M, Caan B, Packer L. Factors associated with oxidative stress in human populations. Am J Epidemiol. 2002;156(3): 274-85.

53. Leszek J, E Barreto G, Gasiorowski K, Koutsouraki E, Aliev G. Inflammatory mechanisms and oxidative stress as key factors responsible for progression of neurodegeneration: role of brain innate immune system. CNS Neurol Disord Drug Targets. 2016;15(3): 329-36.

54. Ezzati M. Methodology for assessment of environmental burden of disease - Annex 4.1 [Internet]. Who.int. 2010 [citado 7 de mayo 2019]. Disponible en: https://tinyurl.com/y6c4pkb3.

55. Leung AA, DaskalopoulouSS, DasguptaK, McBrien $\mathrm{K}$, Butalia S, Zarnke KB, et al. Hypertension Canada's 2017 guidelines for diagnosis, risk assessment, prevention, and treatment of hypertension in adults. Can J Cardiol. 2017;33(5): 557-76. doi: https://doi.org/10.1016/j.cjca.2017.03.005.

56. Tran MT, Mitchell TM, Kennedy DT, Giles JT. Role of coenzyme Q10 in chronic heart failure, angina, and hypertension. Pharmacotherapy. 2001;21(7): 797-806.

57. Mrowka R. Recent Advances in Hypertension Research. Acta Physiol (Oxf). 2019;8: e13295. doi: 10.1111/apha.13295.

58. Kintscher U. The burden of hypertension. EuroIntervention. 2013;9 (Suppl R): R12-15. doi: 10.4244/EIJV9SRA3.

59. Yao EH, Fukuda N, Matsumoto T, Kobayashi N, Katakawa M, Yamamoto C, et al. Losartan improves the impaired function of endothelial progenitor cells in hypertension via an antioxidant effect. Hypertens Res. 2007;30(11): 1119-28. doi: 10.1291/hypres.30.1119.

60. Profumo E, Buttari B, Saso L, Rigano R. Pleiotropic effects of statins in atherosclerotic disease: focus on the antioxidant activity of atorvastatin. Curr Top Med Chem. 2014;14(22): 2542-51. doi: 10.2174/ 1568026614666141203130324.

61. Simic DV, Mimic-Oka J, Pljesa-Ercegovac M, Savic-Radojevic A, Opacic M, Matic D, Simic T. Byproducts of oxidative protein damage and antioxidant enzyme activities in plasma of patients with different degrees of essential hypertension. J Hum Hypertens. 2006;20(2): 149-55. doi: https://doi.org/10.1038/sj.jhh.1001945.

62. Touyz RM, Schiffrin EL. Reactive oxygen species in vascular biology: implications in hypertension. Histochem Cell Biol. 2004;122(4): 339-52. doi: https://doi.org/10.1007/s00418-004-0696-7.

63. Duffy SJ, Gokce N, Holbrook M, Huang A, Frei B, Keaney JF, Vita JA. Treatment of hypertension with ascorbic acid. Lancet. 1999;354(9195): 2048-9. doi: https://doi. org/10.1016/S0140-6736(99)04410-4. 
64. Fotherby MD, Williams JC, Forster LA, Craner P, Ferns GA. Effect of vitamin C on ambulatory blood pressure and plasma lipids in older persons. J Hypertens. 2000;18(4): 411-5.

65. Juraschek SP, Guallar E, Appel LJ, Miller ER 3rd. Effects of vitamin C supplementation on blood pressure: a meta-analysis of randomized controlled trials. Am J Clin Nutr. 2012;95(5): 1079-88. doi:10.3945/ajen.111.027995.

66. Heart Protection Study Collaborative Group: MRC/BHF Heart Protection Study of antioxidant vitamin supplementation in 20,536 high-risk individuals: A randomised placebo-controlled trial. Lancet. 2002;360(9326): 23-33. doi: 10.1016/S0140-6736(02)09328-5.

67. Kim MK, Sasaki S, Sasazuki S, Okubo S, Hayashi M, Tsugane S. Lack of long-term effect of vitamin C supplementation on blood pressure. Hypertension. 2002;40(6): 797-803.

68. Palumbo G, Avanzini F, Alli C, Roncaglioni MC, Ronchi E, Cristofari M, et al. Effects of vitamin $\mathrm{E}$ on clinic and ambulatory blood pressure in treated hypertensive patients: Collaborative Group of the Primary Prevention Project (PPP) Hypertension Study. Am J Hypertens. 2000;13(5 Pt 1): 564-7.

69. Münzel T, Gori T, Bruno RM, Taddei S. Is oxidative stress a therapeutic target in cardiovascular disease? Eur Heart J. 2010 Nov;31(22): 2741-8. doi: 10.1093/eurheartj/ehq396.

70. Heart Outcomes Prevention Evaluation Study Investigators, Yusuf S, Sleight P, Pogue J, Bosch J, Davies R, Dagenais G. Effects of an angiotensin-converting-enzyme, ramipril, on cardiovascular events in high-risk patients. $\mathrm{N}$ Engl J Med. 2000;342(3): 145-53. doi: 10.1056/ NEJM200001203420301.

71. Head GA, Shaw JE, Dunstan DW, Owen N, Magliano DJ, Chadban S, Zimmet P. Hypertension, white-coat hypertension and masked hypertension in Australia: findings from the Australian Diabetes, Obesity, and Lifestyle Study 3. J Hypertens; 2019. doi: 10.1097/ HJH.0000000000002087. [Ahead of print].

72. Schulz E, Gori T, Münzel T. Oxidative stress and endothelial dysfunction in hypertension. Hypertens Res. 2011;34(6): 665-73. doi: https://doi.org/10.1038/hr.2011.39.

73. Dikalova AE, Itani HA, Nazarewicz RR, McMaster WG, Flynn CR, Uzhachenko R, et al. Sirt3 Impairment and SOD2 Hyperacetylation in Vascular Oxidative Stress and Hypertension. Circ Res. 2017;121(5): 564-74. doi:10.1161/ CIRCRESAHA.117.310933.

74. Haidara MA, Yassin HZ, Rateb M, Ammar $\mathrm{H}$, Zorkani MA. Role of oxidative stress in development of cardiovascular complications in diabetes mellitus. Curr Vasc Pharmacol. 2006;4(3): 215-27. doi: https://doi.org/10. 2174/157016106777698469.

75. Kodavanti UP, Schladweiler MC, Ledbetter AD, Watkinson WP, Campen MJ, Winsett DW, et al. The spontaneously hypertensive rat as a model of human cardiovascular disease: evidence of exacerbated cardiopulmonary injury and oxidative stress from inhaled emission particulate matter. Toxicol Appl Pharmacol. 2000;164(3): 250-63. doi: https://doi.org/10.1006/taap.2000.8899.

76. Testa R, Bonfigli AR, Genovese S, De Nigris V, Ceriello, A. The possible role of flavonoids in the prevention of diabetic complications. Nutrients. 2016;8(5). pii: E310. doi: 10.3390/nu8050310.

77. Tanveer A, Akram K, Farooq U, Hayat Z, Shafi A. Management of diabetic complications through fruit flavonoids as a natural remedy. Crit Rev Food Sci Nutr. 2017;57(7): 1411-22. doi: 10.1080/10408398.2014.1000482.

Ciencia y Salud 2019; 3(2): 17-33 • Artículo original 